\title{
Estimation of R0 for the spread of SARS-CoV-2 in Germany from Excess Mortality
}

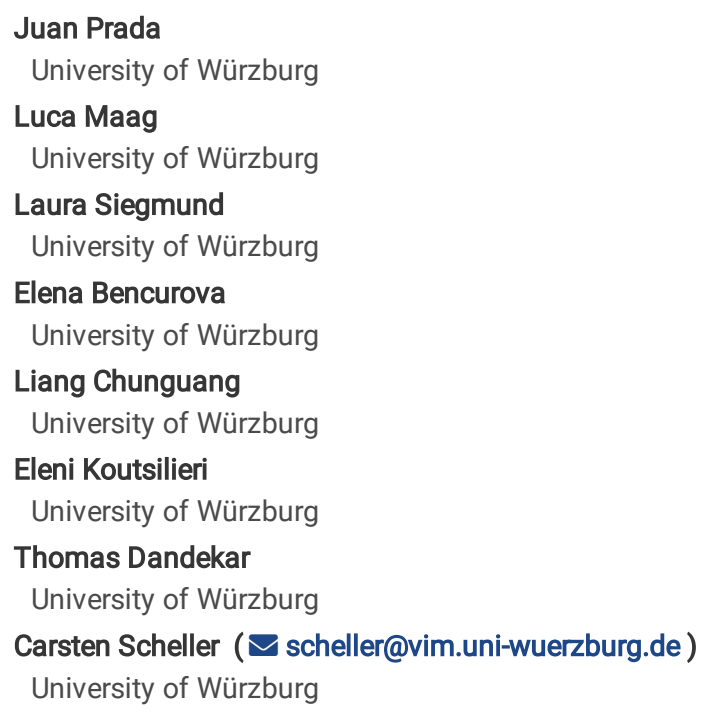




\section{Abstract \\ Background}

For SARS-CoV-2, R0 calculations in the range of 2-3 dominate the literature, but much higher estimates have also been published. Because capacity for PCR testing increased greatly in the early phase of the Covid-19 pandemic, R0 determinations based on these incidence values are subject to strong bias. We propose to use Covid-19-induced excess mortality to determine R0 regardless of PCR testing capacity.

\section{Methods}

We used data from the Robert Koch Institute (RKI) on the incidence of Covid cases, Covid-related deaths, number of PCR tests performed, and excess mortality calculated from data from the Federal Statistical Office in Germany. We determined R0 using exponential growth estimates with a serial interval of 4.7 days. We used only datasets that were not yet under the influence of policy measures (e.g., lockdowns or school closures).

\section{Results}

The uncorrected R0 value for the spread of SARS-CoV-2 based on PCR incidence data was 2.56 (95\% Cl 2.52-2.60) for Covid-19 cases and 2.03 (95\%Cl 1.96-2.10) for Covid-19-related deaths. However, because the number of PCR tests increased by a growth factor of 1.381 during the same period, these R0 values must be corrected accordingly (R0corrected = R0uncorrected/1.381), yielding 1.86 for Covid-19 cases and 1.47 for Covid-19 deaths. The R0 value based on excess deaths was calculated to be 1.34 ( $95 \% \mathrm{Cl} 1.32-1.37)$. A sine-function-based adjustment for seasonal effects of $40 \%$ corresponds to a maximum value of $\mathrm{R} 0_{\text {January }}=1.68$ and a minimum value of $\mathrm{R} 0_{\text {July }}=1.01$.

\section{Discussion}

Our calculations show an R0 that is much lower than previously thought. This relatively low range of R0 fits very well with the observed seasonal pattern of infection across Europe in 2020 and 2021, including the emergence of more contagious escape variants such as delta or omicron. In general, our study shows that excess mortality can be used as a reliable surrogate to determine the R0 in pandemic situations.

\section{Introduction}

The basic replication number (R0) of a virus describes the average number of secondary infections caused by an infected individual in an immunologically still naive population ${ }^{1}$. RO is a key factor in predicting the spread of a virus in a population. It is also used to estimate the proportion of individuals required in a population to achieve herd immunity ${ }^{2}$. In addition, the magnitude of R0 can also be used to predict whether a respiratory virus in temperate climates will develop a seasonal pattern of infection (as observed with influenza viruses and endemic coronaviruses) rather than continuous transmission throughout the year ${ }^{3}$.

R0 is influenced not only by intrinsic characteristics of the pathogen, such as its infectivity and mode of transmission, but also by characteristics of the population under study. For respiratory viruses, there are several such extrinsic characteristics that have a significant impact on the probability of transmission and thus on R0: The density of a population, the number of persons living in a household and their average vulnerability to infections, other social factors that affect the number of close contacts between infected and uninfected persons (e.g., use of public transportation, work laws when ill, etc.), and also the climate of the area in which the population is located ${ }^{4}$.

Based on data from 425 confirmed cases in Wuhan, R0 of SARS-CoV-2 was estimated to be $2.2^{5}$. Another report estimating R0 based on case reports in Wuhan yielded a higher R0 of $5.7^{6}$. This wide range of values is also reflected in a number of other analyses in which R0 was determined to be between 1.95 (WHO estimate) and 6.49 (all reviewed in ${ }^{7}$ ). The German RKI assumes an R0 in the range of 2.8-3.8 ${ }^{8}$ based on systematic reviews ${ }^{9,10,11}$.

All these estimations of RO have in common that they are based on incidences of SARS-CoV-2 infections detected by PCR. These estimations are therefore not only dependent on the characteristics of the population under study, but also on testing strategies (e.g. representative sampling, symptombased testing, contact-based testing of index patients, etc.) as well as rapidly increasing numbers of available and performed tests during the early weeks of the pandemic (at least, if no mathematical corrections for this increase were performed).

Because SARS-CoV-2 infections have led to excess mortality in many countries worldwide ${ }^{12}$, the increase in excess mortality can be used as a surrogate for SARS-CoV-2 infections in order to calculate R0 independent of testing strategies and testing capacity. Here, we determined R0 for SARS-CoV-2 infections in Germany during the early phase of the epidemic in February and March 2020 based on Covid-19-associated excess mortality. For comparison, we also calculated R0 from incidence data of SARS-CoV-2 infections corrected by the increase in test capacities, as well as R0 from incidence data of PCR-confirmed Covid-19-related deaths.

\section{Methods}




\section{Databases}

The number of Covid-19 cases, Covid-19-related deaths and SARS-CoV-2-PCR-tests was accessed from the RKI website ${ }^{13}$. The definition of "Covid-19 case" used here is that of the RKI, which does not use the date of receipt of a positive PCR sample, but rather the date of illness, which in some cases is several days earlier ${ }^{13}$. Excess mortality was calculated from data the of the The Federal Statistical Office ${ }^{14}$. Mobility data was taken from the Apple ${ }^{\mathrm{TM}}$ website $^{15}$. All used datasets can be downloaded as excel file from the supplementary material S1.

\section{Calculation of excess mortality}

To calculate excess mortality per calendar week, the number of weekly deaths ${ }^{14}$ in 2020 was subtracted from the mean of weekly deaths in $2016-2019$. For calculation of "adjusted excess deaths", the excess mortality in calendar week 10 was tared to 0 in all age groups and the values of the following calendar weeks were adjusted accordingly.

\section{Calculation of RO}

$\mathrm{R} 0$ was determined using the R package from Obadia et al. ${ }^{16}$ in R version 3.60. We selected the exponential growth method of the package for calculation of RO. The mean serial interval (average time between successive infection cases) was simulated following a gamma distribution with mean equal to 4.7 ( \pm SD 2.9$)^{17}$. Weekly incidence values of excess mortality were converted to simulated daily incidence values using a gamma distribution. The R-script can be downloaded from the supplementary material S2.

\section{Results}

\section{Determination of the time period that can be used for the calculation of RO.}

The governments of Germany and its states have taken several measures to contain the SARS-CoV-2 epidemic in Germany in early 2020 , including canceling mass events (implemented March 9), closing schools (implemented March 16), closing stores (except grocery stores and pharmacies) and implementing social distancing rules prohibiting personal contact outside the family (implemented March 23) (Fig. 1A). All of these measures, as well as widespread media coverage of the SARS-CoV-2 epidemic in Germany, likely had an impact on the spread of SARS-CoV-2. Therefore, to estimate the value of RO in Germany, it is imperative to include only data from time points that either predate the implementation of these measures or from time points when these measures could not yet have had an impact on the observed parameter used to calculate R0. As shown in Fig. 1A, people in Germany started to reduce their mobility from March 12, i.e., even a few days earlier than social distancing was officially introduced. Because the incubation period between SARS-CoV-2 infection and the onset of Covid-19 symptoms is on average 5-6 days ${ }^{8}$, behavioral changes can lead to an impact on the number of disease cases no earlier than 5-6 days later. For our R0 calculations, we were therefore able to use incidence data of Covid-19 disease cases up to and including March 15 (calendar week 12) without risking that behavioral changes may have had an impact on the R value determined (Fig. 2C).

The German Robert Koch Institute (RKI) provides different epidemiological datasets that can be used for calculations of R0 of SARS-CoV-2 such as daily numbers of detected cases and daily numbers of CoViD-19-related deaths. We fitted the data to a gamma distribution and determined the difference between the peaks of the curves. The mean time between the occurrence of Covid-19 disease cases and Covid-19-related deaths was 25 days (Fig. 1B). Covid-19-related deaths can therefore be used for the determination of R0 at significantly later time points than the occurrence of Covid-19 disease cases without risking of compromising the RO value by behavioral changes. Therefore, to determine R0 from reported Covid-19 deaths (as well as from Covid19-related excess mortality), we used records up to and including April 11 (calendar week 15) (Fig. 2C).

\section{Calculation of RO from incidence data of Covid-19 disease cases and Covid-19 deaths.}

In the initial phase of the pandemic, testing capacities were significantly smaller than the actual number of infections. The steep rise in the number of reported cases during this period might therefore also due in significant part to the sharp increase in the number of SARS-CoV-2 PCR test performed. For our calculations of R0, we use incidence data up to and including calendar week 12 for Covid-19 disease cases and up to and including calendar week 15 for Covid-19 deaths. While data for the number of tests performed are not available for the period before calendar week 11, the RKI provides at least the number of tests performed from week 11 onwards ${ }^{13}$. As depicted in fig. $2 \mathrm{~A}$, a significant increase in the number of tests performed can be observed in the calendar weeks 11 to 13 . To determine what impact this increase in testing numbers had on reported Covid-19 incidences, we determined the growth rate of testing during this period. The growth rate of testing yields an "R0 of tests" of 1.38 (Fig. 2A), meaning that even if the number of infections remained constant during this period of time, an apparent increase of 1.38 in RO would be observed. It follows that RO values from incidence figures must be corrected by this factor.

From the raw incidence data, we obtain an R0 of 2.56 for Covid-19 disease cases and an R0 of 2.03 for Covid-19 death cases (Fig. 2B). However, these values must still be corrected for the growth rate of testing $\left(\mathrm{R}_{\text {corrected }}=\mathrm{R} 0_{\text {uncorrected }} /\right.$ " $\mathrm{R} 0$ of tests"), resulting in a corrected $\mathrm{R} 0$ of 1.86 for Covid-19 disease cases and an R0 of 1.47 for Covid-19-death cases. The R0 value derived from deaths is slightly lower than the R0 value determined from Covid19 incidence values. This may be due to the fact that in the initial phase of the pandemic, severely ill cases (and thus individuals at higher risk of death) were preferentially tested, while milder and asymptomatic cases were increasingly included in testing as the testing capacity expanded. Such a change in testing strategy inevitably introduces a bias toward higher RO values when calculated from Covid-19 incidence data compared with Covid-19 death data. 
However, even if we correct the incidence values for test capacity dynamics, this way of determining R0 still remains subject to many uncertainties: First, the exact numbers of tests performed in the first weeks of the pandemic were not collected for Germany, so that an accurate estimate of the dynamics of testing capacity is not possible. Second, the incidence data do not come from representative samples in the general population, but mainly from symptomatic patients and persons with whom they came into contact. Therefore, this dataset contains a disproportionate number of infections from nursing homes and hospitals, where symptomatic infections are overrepresented and where transmission probabilities are most likely different from what would be expected in the general population. Therefore, the R0 values calculated above are unlikely to be representative of the spread of the virus in the general population.

\section{Calculation of RO from Excess mortality}

To address this problem, we also determined R0 based on excess mortality data in Germany during early 2020. The Federal Statistical Office of Germany lists all deaths that occur in Germany, regardless of their cause ${ }^{14}$. Because SARS-CoV-2 infection has led to increased excess mortality in many countries, these data can be used as surrogate markers for the spread of SARS-CoV-2 infections. And because excess mortality is independent of the number or strategy of SARS-CoV-2 testing, it provides a representative picture for the spread of infections in the general population.

Figure $3 \mathrm{~A}$ shows the incidence of deaths with confirmed SARS-CoV-2 infection. The data set shown here is the same as that in Figure $2 \mathrm{~B}$, but this time as weekly incidence and subdivided into different age groups. The (uncorrected) R0 value here is 1.95 , similar to 2.03 from figure $2 \mathrm{~B}$. From this figure, it can be seen that the peak of Covid-19 related mortality is between calendar week 10 and 20 . Figure $3 \mathrm{~B}$ shows excess mortality (in relation to average weekly deaths in 2016-2019) in the different age groups, and one can see a parallel trend to the confirmed Covid-related deaths between calendar weeks 10 and 20. Based on the respective values of calendar week 10, from which an increase in excess mortality is observed in all Covid-19 relevant age groups, we plotted the change in all values in Figure 3C. From this adjusted excess mortality, we obtained an R0 of 1.34 ( $95 \% \mathrm{Cl} 1.32-1.37)$ for the sum of all age groups for the spread of SARS-CoV-2 in the general population in Germany.

\section{Influence of Influenza-related excess deaths on Covid-19 related excess deaths}

Due to the lack of representative measurements, Covid-19 related excess mortality is the only infection parameter that is free from bias due to changes in testing strategy or testing numbers. However, excess mortality data are subject to other confounding factors that may have an impact on the calculation of R0: The Covid-19 pandemic reached Germany at a time when seasonal influenza activity in Germany was already subsiding (see Fig. 4C). Influenzarelated excess mortality and Covid-19 related excess mortality are therefore superimposed in the total excess mortality data sets. If influenza mortality had been significantly elevated in 2020 compared with previous years (2016-2019), this could mask Covid-19 related effects, particularly if influenza mortality rates that were already falling again coincided with an incipient increase in Covid-19 mortality rates. However, a look at mortality in previous years shows that influenza-related mortality in Germany in 2020 was significantly lower compared with previous years because of the two exceptionally strong influenza years 2017 and 2018 (Fig. 4A). As a result, under-mortality was observed in Germany in calendar weeks 10-14 compared with previous years, rather than excess-mortality (Fig. 4B). Thus, if there was an effect of influenza-related deaths on the calculation of R0 for Covid-19 infections, it was one that resulted in an overestimate of R0 rather than an underestimate. Thus, the R0 calculated here of 1.34 ( $95 \% \mathrm{Cl} 1.32-1.37)$ should be regarded as a maximum value, whereas the actual RO of SARS-CoV-2 infections in Germany is likely to be even lower.

\section{Discussion}

The early SARS-CoV-2 infection spread in Germany with an R0 of 1.34 (95\% Cl 1.32-1.37). This value is much lower than what had been expected based on R0 determinations from the literature, where values between 2-3 became consensus ${ }^{7,8}$. Although the German RKI has not published an R0 estimation for Germany, it provided daily estimations of R based on a four-day-period. These daily R values during the first two weeks of March 2020 were in the range of 2.2-3.2 ${ }^{18}$. Based on the reporting data for positive PCR results from the "our world in data" database of Oxford University, an R0 of 3.37 was determined for Germany ${ }^{19}$, but these calculations used PCR reporting data rather than data for Covid-19 disease cases and therefore are not fully comparable with the calculations from the RKI. The discrepancies between these high values and the rather low R0 estimates in our manuscript are primarily due to the fact that in these earlier estimations the R0 values were not corrected by a factor accounting for the substantial increase in test capacity during this period. If we use our uncorrected R0 estimate based on Covid-19 case numbers for comparison (R0 $=2.56$, Fig. $2 \mathrm{~B})$, it is in the same order of magnitude as the values calculated by the RKI.

A high R0 of the order of 3 would likely have resulted in a lack of seasonal progression, as a seasonal effect was estimated to reduce R0 by only $40 \%$ based on observations in endemic coronaviruses ${ }^{3}$. Accordingly, health authorities expected an unrestrained spread of the virus for Germany, whereupon a series of policy measures were adopted aiming to actively reduce the incidence of infection. In retrospect, however, a clearly seasonal occurrence is evident not only for Germany, but also for all other countries in temperate climates, in particular also for Sweden, where hardly any measures have been taken to contain the spread of SARS-CoV-2 in the general population ${ }^{12}$.

The seasonal effect on R0 can be approximated as a sinusoidal pattern with a maximum in January as the coldest month in Germany and a minimum in July as the warmest month, with an approximate $40 \%$ reduction in July compared to January ${ }^{3}$ (Fig. 5 , dotted line). As our calculation of R0 was based on the infection situation in March 2020, it can be expected that the R0 value determined in this way is about $20 \%$ lower than the maximum value that would be reached in January if the pandemic would have reached Germany earlier. According to this model, R0 determined in March with a value of $\mathrm{RO}_{\text {March }}=1.34$ would reach its maximum in January with a value of $\mathrm{R} 0_{\text {January }}=1.68$ and fall to a minimum of R0 July $=1.01$ in July $(F i g .5 \mathrm{~A})$. 
Herd immunity is dependent on R0 (herd immunity $=(1-1 / R 0))$, and therefore also fluctuates seasonally. Thus, herd immunity against the original SARSCoV-2 strain would oscillate between $40 \%$ in January and below $1 \%$ in July (Fig. 5B). This explains why the number of SARS-CoV-2 infections in summer 2020 not only declined again in "lockdown" countries such as Germany (and remained low in these countries even after the suspension of policy measures during the summer), but also why the same seasonal pattern was observed in countries with little or no countermeasures against SARS-CoV-2 12 .

With a seasonal increase in the threshold of herd immunity, the following winter (2020/2021) fueled again the spread of SARS-CoV-2 Germany, until infection numbers dropped again in spring 2021. At this time, about 3.8 million SARS-CoV-2 infections have been reported to the RKI, corresponding to $4.5 \%$ of the population (May 2021) ${ }^{20}$ and a serological survey of blood donors revealed a seropositivity rate of $14 \%$ in April 202121 , showing a substantial underestimation of SARS-CoV-2 infections from PCR-data alone. Together with the enrollment of the Covid-vaccine campaign, immunity in the German population has reached up to $40 \%$ by end of May 2021, and that coincided with the emergence of the delta variant, which can now be interpreted as an escape variant that overcame the $40 \%$ herd immunity restrictions of the original SARS-CoV-2 strains by higher contagiousness (and therefore also a higher R0).

Since the population in Germany was no longer naïve towards SARS-CoV-2 during summer of 2021 when the delta variant began its expansion in Germany, it is not possible to determine an RO for this variant. However, the RKI calculates daily $\mathrm{R}_{\mathrm{e}}$ values based on a 7-day period for Germany, and the values for the delta variant reached 1.3 during July/August $2021^{22}$. With a seasonal variation of $40 \%$, this value corresponds to a theoretic maximum in December with an $\mathrm{R}_{\mathrm{e}}=2.2$, translating into a $55 \%$ threshold for winter herd immunity.

Approximately $65 \%$ of the population in Germany has now been vaccinated against SARS-CoV-2 (by end of October 2021). Recent studies show that vaccine efficiency to protect from infection is poor: a cohort study from September 2021 found that Covid vaccines are only $35 \%$ protective against infection ${ }^{23}$, and immunity is expected to decline even more over time. The proportion of the population in Germany already immune to SARS-CoV-2 infection can therefore hardly be estimated without serological data from representative samplings.

The concept of herd immunity in respiratory pathogens such as coronaviruses does not imply permanent protection of the population against seasonal reemergence of these pathogens, since the immunity achieved may decrease over time, especially in asymptomatically infected patients ${ }^{24}$. Instead, the achievement of herd immunity in respiratory viruses leads to a strong selection pressure for escape mutations (classical immune escape or increased contagiousness), which can then give rise to new waves of infection ${ }^{25}$. For this reason, respiratory viruses such as influenza- or coronaviruses remain endemic, despite broad immunity, which will probably also be the case for SARS-CoV-2.

\section{Conclusion}

Our study shows that the RO value of SARS-CoV-2 can be calculated from excess mortality data. We also introduce here the concept of a seasonally adjusted $\mathrm{RO}$ value, which should be reported as a range ( $\mathrm{R} \mathrm{J}_{\text {January }}$ - $\mathrm{R} \mathrm{J}_{\text {July }}$ ) rather than a static value. We determined an $\mathrm{R} 0$ value of 1.34 for infections in March $2020\left(\mathrm{R}_{\text {March }}=1.34\right)$, corresponding to a seasonal range of $\mathrm{R} 0_{\text {January }}=1.68$ and a minimum in July $\left(\mathrm{R} 0_{\mathrm{July}}=1.01\right)$. This rather low range of $\mathrm{R} 0$ values is much more consistent with observations of pandemic progression than many earlier and much higher estimates of the R0 value. The massive expansion of testing capacity in the early phase of the pandemic, combined with changes in testing strategy, was a major cause of the overestimation of the RO value. Excess mortality can be determined independently of SARS-CoV-2 testing capacity in many countries, and therefore can be a valuable tool in future pandemics to provide reliable values for the rate of spread of an emerging pathogen in a population when representative samples of pathogen spread are not available.

\section{Declarations}

\section{Acknowledgements:}

For the preparation of this manuscript, we used the online version of DeepL translator ${ }^{24}$ and Mendeley Reference Manager ${ }^{25}$.

\section{Supplementary material:}

S1 Database

S2 R Script

S3 Readme

\section{Funding}

We acknowledge Land of Bavaria contribution to DFG project 324392634-TRR 221/INF (TD) and DFG project 374031971-TRR 240/INF (TD).

\section{Competing interests:}

Authors declare that they have no competing financial or non-financial interests. 


\section{Author contributions:}

Conceptualization: CS

Methodology: JPP, TD, CS

Investigation: JPP, LEM, LS, EB, LC, EK, TD, CS

Visualization: JPP, LEM, LS, EB, LC,

Funding acquisition: TD, CS

Project administration: TD, CS

Supervision: EK, TD, CS

Writing - original draft: JPP, CS

Writing - review \& editing: JPP, LEM, LS, EB, LC, EK, TD, CS

\section{References}

1. Dietz K. The estimation of the basic reproduction number for infectious diseases. Stat Methods Med Res. Published online 1993. doi:10.1177/096228029300200103

2. Anderson RM, May RM. Vaccination and herd immunity to infectious diseases. Nature. Published online 1985. doi:10.1038/318323a0

3. Kissler SM, Tedijanto C, Goldstein EM, Grad YH, Lipsitch M. Projecting the transmission dynamics of SARS-CoV-2 through the post-pandemic period. Science (80-). Published online 2020. doi:10.1101/2020.03.04.20031112

4. Delamater PL, Street EJ, Leslie TF, Yang YT, Jacobsen KH. Complexity of the basic reproduction number (R0). Emerg Infect Dis. Published online 2019. doi:10.3201/eid2501.171901

5. Li Q, Guan X, Wu P, et al. Early Transmission Dynamics in Wuhan, China, of Novel Coronavirus-Infected Pneumonia. N Engl J Med. Published online 2020. doi:10.1056/nejmoa2001316

6. Sanche S, Lin YT, Xu C, Romero-Severson E, Hengartner N, Ke R. High Contagiousness and Rapid Spread of Severe Acute Respiratory Syndrome Coronavirus 2 - Volume 26, Number 7-July 2020 - Emerging Infectious Diseases journal - CDC. Emerg Infect Dis. Published online 2020.

7. Liu Y, Gayle AA, Wilder-Smith A, Rocklöv J. The reproductive number of COVID-19 is higher compared to SARS coronavirus. $J$ Travel Med. 2020;27(2):1-4. doi:10.1093/jtm/taaa021

8. RKI Coronavirus Steckbrief. Accessed August 28, 2021. https://www.rki.de/DE/Content/InfAZ/N/Neuartiges_Coronavirus/Steckbrief.html;jsessionid=635EC49A76E4BD9C2F02941EB844EA6E.internet112? $\mathrm{nn}=13490888 \#$ doc13776792bodyText5

9. Wei Y, Wei L, Liu Y, et al. A systematic review and meta-analysis reveals long and dispersive incubation period of COVID-19. medRxiv. Published online 2020. doi:10.1101/2020.06.20.20134387

10. Yang L, Dai J, Zhao J, Wang Y, Deng P, Wang J. Estimation of incubation period and serial interval of COVID-19: Analysis of 178 cases and 131 transmission chains in Hubei province, China. Epidemiol Infect. Published online 2020. doi:10.1017/S0950268820001338

11. Linton N, Kobayashi T, Yang Y, et al. Incubation Period and Other Epidemiological Characteristics of 2019 Novel Coronavirus Infections with Right Truncation: A Statistical Analysis of Publicly Available Case Data. J Clin Med. Published online 2020. doi:10.3390/jcm9020538

12. Vestergaard LS, Nielsen J, Richter L, et al. Excess all-cause mortality during the COVID-19 pandemic in Europe - preliminary pooled estimates from the EuroMOMO network, March to April 2020. Eurosurveillance. Published online 2020. doi:10.2807/1560-7917.ES.2020.25.26.2001214

13. Robert Koch Institut. Täglicher Lagebericht des RKI zur Coronavirus-Krankheit-2019 (COVID-19). Robert Koch Inst. 2020;2019:1-7. https://www.rki.de/DE/Content/InfAZ/N/Neuartiges_Coronavirus/Situationsberichte/Archiv.html

14. DeStatis. https://www.destatis.de/DE/Themen/Querschnitt/Corona/Gesellschaft/bevoelkerung-sterbefaelle.html

15. Apple Mobility Data. Accessed August 28, 2021.

16. Obadia T, Haneef R, Boëlle PY. The R0 package: A toolbox to estimate reproduction numbers for epidemic outbreaks. BMC Med Inform Decis Mak. 2012;12(1). doi:10.1186/1472-6947-12-147

17. Nishiura H, Linton NM, Akhmetzhanov AR. Serial interval of novel coronavirus (COVID-19) infections. Int J Infect Dis. 2020;93:284-286. doi:10.1016/j.ijid.2020.02.060

18. Rki RK. 17 Epidemiologisches 2020 Bulletin. 2020;(April).

19. Spouge JL. A comprehensive estimation of country-level basic reproduction numbers ROfor COVID-19: Regime regression can automatically estimate the end of the exponential phase in epidemic data. PLoS One. 2021;16(7 July):1-14. doi:10.1371/journal.pone.0254145 
20. Robert Koch Institut. Bericht zu Virusvarianten von SARS-CoV-2 in Deutschland. Robert Koch Inst. 2021;2:1-19. https://www.rki.de/DE/Content/InfAZ/N/Neuartiges_Coronavirus/DESH/Bericht_VOC_2021-06-23.pdf

21. RKI SeBluCo Study. Accessed July 29, 2021. https://www.rki.de/DE/Content/InfAZ/N/Neuartiges_Coronavirus/Projekte_RKI/SeBluCo_Zwischenbericht.html

22. Anna F, Goyard S, Lalanne Al, et al. High seroprevalence but short-lived immune response to SARS-CoV-2 infection in Paris. Eur J Immunol. 2021;51(1):180-190. doi:10.1002/eji.202049058

23. Planas D, Veyer D, Baidaliuk A, et al. Reduced sensitivity of SARS-CoV-2 variant Delta to antibody neutralization. Nature. 2021;596(7871):276-280. doi:10.1038/s41586-021-03777-9

24. DeepL. https://www.deepl.com/de/translator

25. Mendeley Reference Manager. Accessed August 28, 2021. https://www.mendeley.com/download-desktop-new/

\section{Figures}

\section{Figure 1}

Identifying SRAS-CoV-2 datasets unaffected by policies or behavioral changes for estimating R0. A: Mobility data (driving) for Germany, provided by AppleTM Inc. 15 (driving: red; transit: blue; walking: green). The first change in mobility trends is observed for March 13. B: Data provided by RKI for Covid-cases (black) and Covid-19-related deaths (red) were fitted by gamma distribution. The maxima of the two curves are 25 days apart. C: Graphical representation of the date up to which data from Covid-19 disease cases or Covid-19 death cases can be used to determine R0 without affecting the outcome through policy actions or societal responses.

\section{Figure 2}

Calculation of R0 from Covid-19 disease incidence numbers and Covid-19 related deaths. A: Data reported by the RKI for the number of performed SARACoV-2 PCR-tests. B: Data reported by the RKI for Covid-19-cases (blue symbols, left y-axis) and Covid-19 related deaths (red symbols, right $y$-axis). A,B: Date were fitted to an exponential growth curve with a serial interval of 4.7 ( \pm SD 2.9 ) to calculate R0. Dotted lines in B represent the dates for political interventions (03/09/2020 cancellation of mass events, 03/16/2020 closing of schools, 03/23/2020 closing of shops and social distancing). Dark blue and dark red symbols represent data points that were considered for the determination of R0, light blue and light red symbols represent later data points that were not considered for the calculation.

\section{Figure 3}

Calculation of R0 from excess mortality. A: Covid-19 related deaths as weekly incidence in different age groups. B: Excess deaths in 2020 in different age groups based on comparison with average weekly deaths in 2016-2019. C: Excess deaths presented in B, but adjusted to 0 for week 10 in each age group, so that relative changes related to Covid-19 become better visible.

\section{Figure 4}

Comparison of Influenza-related and Covid-19 related mortality in Germany. A: Daily deaths in Germany from 2016 to 2021 (data taken from the Federal Statistical Office of Germany). B: Comparison of daily number of deaths in 2020 (red line) with average number of daily deaths in $2016-2019$ (blue line). C: Positive rate for influenza infections in Germany for calendar weeks 1-20 from 2016-2020 (data taken from the RKI influenza survey).

\section{Figure 5}

Seasonal influence on R0 and herd immunity. A: The seasonal effect on R0 can be assumed as a sine function with a maximum in January and a $40 \%$ lower minimum in July (dashed line, right $y$-axis). This translates into an oscillating R0 with a maximum in January (of R0January $=1.68$ ) and a minimum in July (ROJuly = 1.01) (blue line, left y-axis). RO calculations are based on the values calculated for March (R0March = 1.34) (blue dot) B: Herd immunity similarly to RO oscillates in a seasonal pattern.

\section{Supplementary Files}

This is a list of supplementary files associated with this preprint. Click to download.

- datasheetR0.xlsx 
- Scriptsupplemental.r

- SuplementalmateriaIREADME.docx

Page $8 / 8$ 